Laura Sánchez Carrasco

\title{
A ambos lados del Atlántico Dos fábricas de Kevin Roche y John Dinkeloo para Cummins Engine Company
}

\author{
Palabras clave: fábrica, Kevin Roche, John Dinkeloo, Commins Engine Company, \\ arquitectura industrial, siglo XX, Darlington, Walesboro.
}

Dentro de la trayectoria del estudio KRJDA, ambos edificios se plantearon como nuevas oportunidades para investigar la arquitectura de los espacios industriales. Representarian la búsqueda del style for the job heredado de su maestro, Eero Saarinen, quien ya habia colaborado con anterioridad con el cliente de las fábricas, J. Irwin MillerEn, en varios proyectos en Columbus, Ohio. El objetivo es revisar y comparar los conceptos que definen estos dos proyectos construidos para el mismo cliente pero en paises diferentes y con requerimientos funcionales distintos. En la planta de Darlington la cuestión se centrará fundamentalmente en sus soluciones constructivas, mientras que en la de Walesboro se reformuló la tipología y el planteamiento funcional de un edificio industrial. Darlington posee grado II de protección desde 1993 y Walesboro ha sido remodelada respetando el espíritu inicial, mereciendo ambas un lugar destacado en el patrimonio industrial del siglo XX.

Kevin Roche (en primer plano) y John Dinkeloo (en el centro de la imagen) en el estudio, delante de un dibujo del Air Force Museum. Fotografia aparecida en la revista Fortune en enero de 1966.
Laura Sánchez

Carrasco

Arquitecta por la Escuela Técnica Superior de Arquitectura de Madrid 2007. Master en Análisis, Teoría e Historia de la Arquitectura por la Escuela Técnica Superior de Arquitectura de Madrid, 2014. Actualmente realizando la tesis doctoral en el Departamento de Composición de la ETSAM bajo el título: "Kevin Roche John Dinkeloo and Associates. Ideas que sustentan su obra arquitectónica." a empresa Cummins Engine Company -ha mostrado siempre un intenso vínculo con la arquitectura contemporánea y no es casual que la ciudad de Columbus, Indiana, donde tiene su sede, se haya convertido en una comunidad rica en ejemplos de la mejor arquitectura moderna americana de mediados del siglo XX. El origen de todo esto se halla en la iniciativa de Joseph Irwin Miller, director de la compañia entre 1934 y 1977, al crear la Cummins Foundation. ${ }^{1}$

La apuesta de Miller por la arquitectura contemporánea también tuvo un gran impacto en la propia empresa Cummins, que en la actualidad cuenta con un interesante patrimonio arquitectónico repartido por el mundo. En él se destacan dos de sus fábricas construidas en las décadas de los años sesenta y setenta, dos ejemplos fundamentales de la arquitectura industrial de la segunda mitad del s. XX: la fábrica de Darlington, Inglaterra (1966) y la planta de Walesboro (1975) en Columbus, Indiana.

Entre todas las plantas manufactureras que posee la empresa en los distintos países donde opera, se han elegido estos dos ejemplos porque se considera que abrieron nuevos caminos dentro el discurso de la arquitectura industrial desde dos puntos de vista distintos. Además, ambas fábricas tienen la peculiaridad de haber sido proyectadas por los mismos arquitectos, Kevin Roche John Dinkeloo and Associates (KRJDA en adelante) cuando los dos socios aún se encontraban en activo. ${ }^{2} \mathrm{El}$ estudio KRJDA destacaba en aquellos años como una de las firmas más ocupadas y reconocidas del país y en estos dos proyectos se

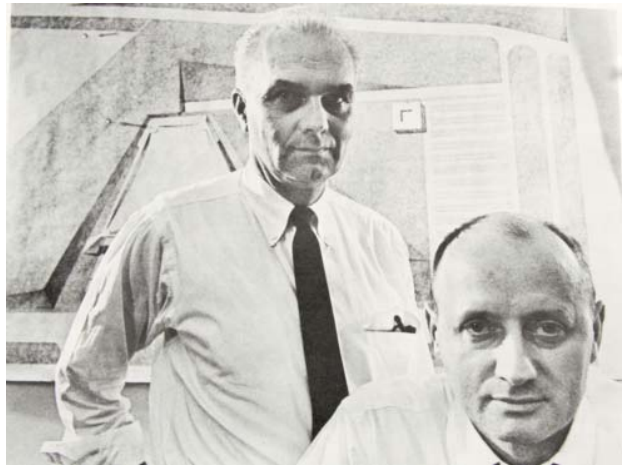

observan soluciones a algunas de las inquietudes que definieron el trabajo del equipo en esos años. Sin embargo, no existe ningún texto anterior que relacione estas dos fábricas.

Ambos edificios se plantearon como nuevas oportunidades para investigar la arquitectura de los espacios industriales pero, como ha sido habitual en la trayectoria de KRJDA, cada uno de ellos se resolvió de manera muy distinta, según las necesidades de cada proyecto. En este sentido, se considera que estas dos fábricas representan la búsqueda del "style for the job"3 que heredaron de su maestro, Eero Saarinen.

Tanto el trabajo de Kevin Roche y John Dinkeloo, como anteriormente el de Eero Saarinen, fue acusado repetidamente de "estilisticamente incoherente" 4 por las publicaciones especializadas. Esto, lejos de ser un problema para ambas oficinas, suponía la esencia de su pensamiento arquitectónico. Cada edificio debía aportar una solución concreta y adecuada a los 
Portada de la revista Esquire con J. Irwin Miller bajo el título

"This man ought to be the next president of the United States". Octubre de 1967. problemas a los que se enfrentaba. Entre ellos se encontraba la relación con el entorno o la solución de aspectos funcionales, pero también, y casi más importante, el interés por reflejar el espíritu de la época utilizando los materiales y las nuevas técnicas de las que se dispusiesen así como resolviendo cuestiones sociales propias del momento, como se verá fundamentalmente en el ejemplo de Walesboro.

El objetivo reside, por tanto, en revisar y comparar los conceptos que definen estos dos proyectos, construidos para el mismo cliente y por los mismos arquitectos pero en dos países diferentes y con requerimientos funcionales distintos.

En el caso de la planta de Darlington, la cuestión se concentra fundamentalmente en el estudio de las soluciones constructivas mientras que en la fábrica de Walesboro, se pretende exponer el trabajo de los arquitectos para reformular la tipología y el planteamiento funcional de los edificios de carácter industrial.

\section{Cummins Engine Company}

La historia de la empresa Cummins Engine Company comienza en 1919, cuando Clessie Lyle Cummins (n. 1888) encuentra el respaldo del banquero de Columbus, William G. Irwin (n. 1866) para desarrollar tecnología relacionada con los motores diesel

Desde sus inicios, la ambición de la firma fue crear una corporación que sobrepasase los limites locales para convertirse en un referente a nivel mundial. En un principio, el camino no fue sencillo y la compañía no obtuvo beneficios hasta los años treinta, momento en el que inició un ligero crecimiento. 5 Pero la reconversión industrial posterior a la Segunda Guerra Mundial y la capacidad empresarial de J. Irwin Miller, sobrino nieto de William G. Irwin, guiaron a la empresa hacia un liderazgo internacional que mantuvo durante cuatro décadas y que permitió que la compañía iniciara sus contribuciones filantrópicas así como inversiones patrimoniales en la propia empresa.

Las biografias de Miller dibujan a un hombre de gran capacidad intelectual, innovador, moderno e involucrado con su comunidad. Tras un breve contacto con la arquitectura contemporánea en la Universidad de Yale ${ }^{6}$ inició el cambio de imagen de la ciudad de Columbus. El primer edificio moderno construido en la ciudad fue la First Christian Church, ${ }^{7}$ encargada a Eliel Saarinen y finalizada en 1942. Durante las

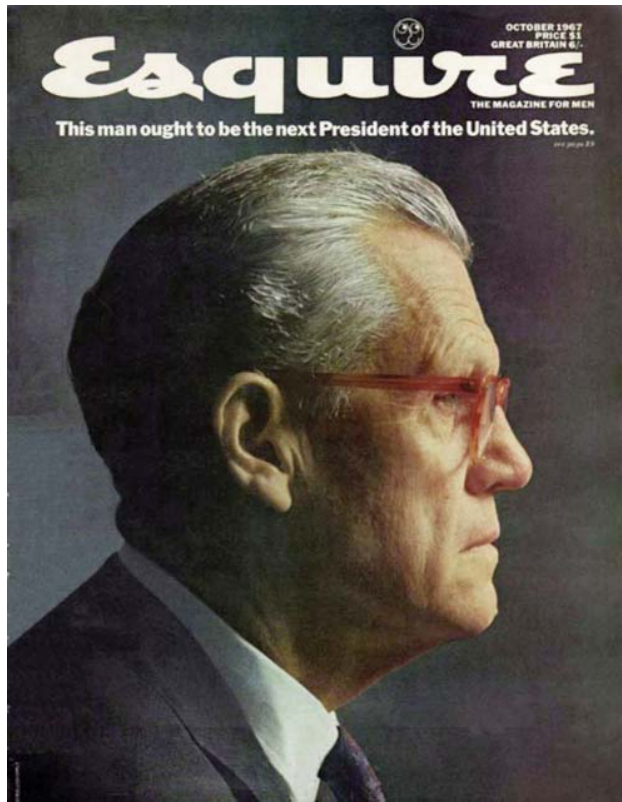

obras de esta iglesia, Miller conoció a Eero Saarinen, que en esos momentos colaboraba con su padre bajo el nombre de Saarinen and Saarinen, Tras la muerte de Eliel Saarinen en 1950, Eero construiria tres proyectos en Columbus: la sede del Irwin Union Bank (1954), la North Christian Church (1964) y la Casa Miller (1957).

En este último proyecto se inició la relación profesional entre Kevin Roche y J. Irwin Miller. Roche trabajaba en Eero Saarinen and Associates desde 1950 y en 1957 ya era uno de sus colaboradores más cercanos. La relación entre Miller y Roche fue buena desde el inicio y se prolongaria hasta el fallecimiento del empresario en 2004. Las declaraciones públicas del propio Miller no dejan lugar a dudas sobre esta sintonía, y es especialmente notoria en dos escritos concretos, fechados después de haber colaborado juntos en las fábricas que protagonizan este artículo. El primer texto es el prefacio para la primera monografia del estudio 8 y el segundo, una carta muy extensa defendiendo la conveniencia de que el American Institute of Architects otorgase su Medalla de Oro a Kevin Roche en 1993, como finalmente sucedió. En esa carta, Miller valoraba que, desde que se conocieron, "Kevin Roche demostró dos características que todavía definen su trabajo: su voluntad de cambiar su diseño cuando claramente no le satisface al cliente, y su determinación para no aceptar ningún diseño que no le satisfaga a él". 9

El primer encargo directo de J. Irwin Miller a Kevin Roche fue la fábrica que la empresa Cummins quería construir en Darling- 
Imagen de época de la fábrica Cummins en Darlington. Imagen aparecida en Architectural Design en agosto de 1967. ton, Inglaterra, poco tiempo después del fallecimiento de Eero Saarinen. ${ }^{10}$

\section{Cummins Engine Company nents Plant, Darlington (1966)}

Compo-

A principios de los años sesenta, Darlington era un área económicamente deprimida del norte de Inglaterra. El hecho de que la empresa Cummins fijara una de sus fábricas en un lugar tan poco representativo del país se debió a un programa de desarrollo industrial impulsado por el gobierno. ${ }^{11} \mathrm{El}$ condado de Durham, en el que se encuentra la ciudad de Darlington, habia sido tradicionalmente un parque industrial dedicado a iniciativas de capital público, fundamentalmente aquéllas relacionadas con la extracción de carbón, que en esos años había perdido atractivo para cualquier otro tipo de inversión empresarial.

Una vez garantizados una serie de beneficios fiscales e impositivos, la empresa americana consideró que Darlington podría ser un buen lugar para fabricar cuatro de sus componentes clave: inyectores de combustible, bombas de combustible, compresores de aire y amortiguadores de vibraciones.

Conviene detenerse un momento a explicar que, aunque aquí sólo se va a tratar de un edificio, en realidad se construyeron dos fábricas de manera casi simultánea y en el mismo lugar. La primera (y protagonista de este artículo) se encargó a $\mathrm{KRJDA}^{12} \mathrm{y}$, en un principio, James Cubitt and Partners ${ }^{13}$ serian los arquitectos locales. La segunda fábrica se denominaba

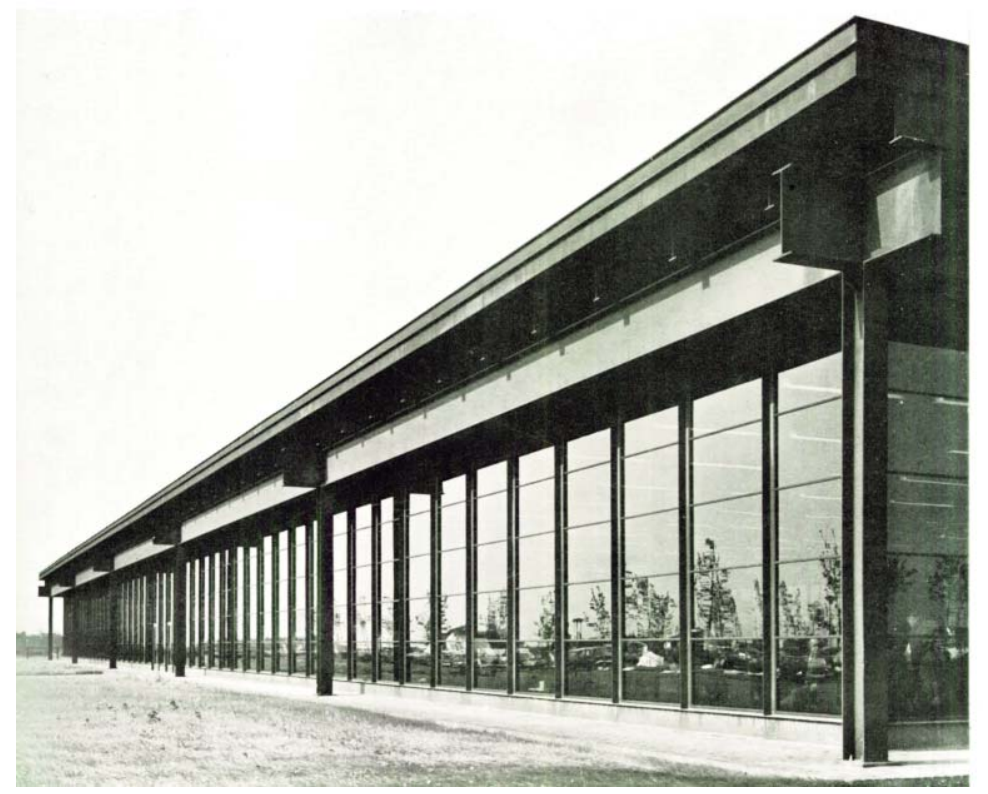

Chrysler-Cummins ya que sería una planta para desarrollar los intereses comunes de ambas empresas, fundamentalmente la ingeniería diesel que Chrysler utilizaba en sus Dodge's Trucks. En este caso, KRJDA también fue el estudio elegido y de nuevo James Cubitt and Partners serian los arquitectos locales asociados. Pero la autoría de esta última fábrica siempre ha generado confusión. La correspondencia entre John Dinkeloo y Fello Atkinson, ${ }^{14}$ el arquitecto y socio de James Cubitt and Partners encargado de esta obra, acredita que KRJDA trabajó en el diseño de este edificio hasta que éste estuvo muy avanzado, pero en ningún momento aparece en sus listas de obras.

Aunque KRJDA no reclama ninguna autoría sobre esta fábrica, sí realizaron planos de proyecto y mantuvieron decisiones durante la obra, puede que favorecidas por la proximidad de ambas plantas. ${ }^{15}$ Pero es cierto que en algunas publicaciones sí que se les menciona como autores o, al menos, como arquitectos asociados de James Cubitt and Partners.

Lo que provoca esta confusión es que, finalmente, KRJDA no terminase las obras. Esto se debió a dos circunstancias. La primera, la escasa afinidad entre ambos estudios de arquitectura. Durante el primer trimestre de 1964, la correspondencia entre Dinkeloo y Atkinson es continua y constantemente se aprecia la disconformidad del arquitecto americano con las decisiones que los arquitectos locales estaban tomando en la obra. Este desacuerdo se evidenciaba tanto respecto a las soluciones técnicas como a los acabados interiores. La segunda razón responde a la urgencia que tenía la propiedad por terminar la fábrica Chrysler-Cummins en el tercer trimestre de 1964, y la imposibilidad del estudio americano de ceñirse a esa fecha. ${ }^{16}$

Por todo lo explicado anteriormente, este artículo descarta el análisis de la fábrica Chyrsler-Cummins y se centra en la planta de componentes Cummins, la que tiene autoría reconocida por el estudio.

En este caso, tanto la propiedad como los arquitectos coincidían en el escaso interés que tenía levantar un hito arquitectónico $\mathrm{y}$, por el contrario, ambos pensaban que lo más acertado era construir una fábrica que cumpliera tres condiciones: ser económica, de calidad y que exportase "la mejor tradición americana" en cuanto a su atractivo conocimiento tecnológico. ${ }^{17}$ 


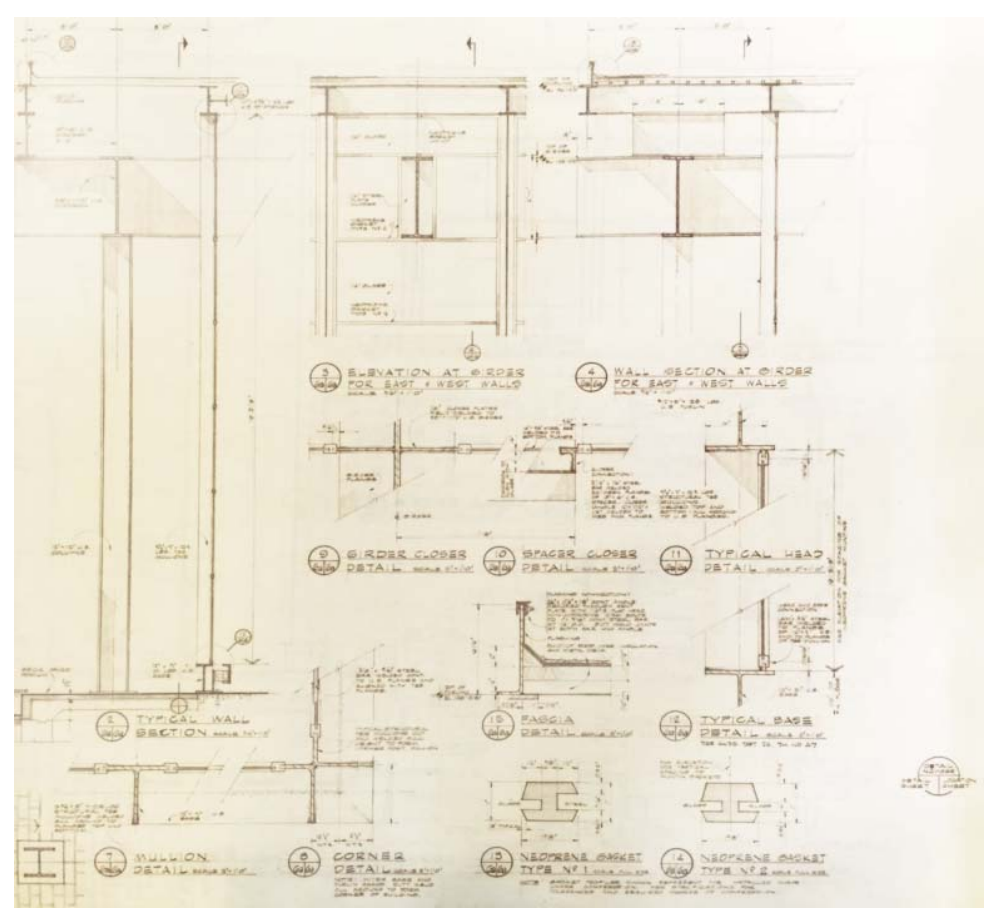

Extracto de plano original de proyecto donde se aprecian los principales nudos de estructura, las uniones de vidrio y las juntas de neopreno. Kevin Roche John Dinkeloo and Associates, MS 1884, Biblioteca de Manuscritos y Archivos, Universidad de Yale.

Esquema de solución estructural y constructiva de la fábrica Cummins en Darlington. Dibujo de la autora.
Para cumplir la primera premisa, el estudio propuso una planta sencilla y trabajó sobre la idea de gran contenedor, "algo muy, muy directo" 18 en palabras del propio Roche.

Para conseguir el estándar de calidad por el que KRJDA ha destacado habitualmente, el estudio decidió prescindir de arquitectos locales y trasladar a Inglaterra a un arquitecto contratado por ellos mismos. Así se lo comunicó John Dinkeloo a Fello Atkinson el 22 de Abril de 1964 ya que, como se ha comentado anteriormente, Cubitt and Partners figuraban en un principio como arquitectos asociados y ya habian desarrollado parte del proyecto juntos. El arquitecto elegido para desplazarse hasta las obras fue John Calvert. Por último, el espíritu de innovación de la sociedad americana lo transmitieron a través de decisiones constructivas y materiales que se explicarán con detalle posterior- mente ya que se consideran los aspectos más destacables de este proyecto.

Los arquitectos decidieron que el protagonismo de esta obra se concentrase en los materiales y en las soluciones constructivas. Uno de sus principales méritos reside en ser el primer edificio en Inglaterra que se construye con acero cor-ten y juntas de neopreno estructurales entre vidrios, dos innovaciones que provienen de la transferencia tecnológica practicada por Eero Saarinen desde los años cincuenta y que Roche y Dinkeloo continuaron posteriormente. De hecho, Saarinen fue el primero en utilizar el acero cor-ten y las juntas de neopreno en la construcción a nivel mundial. ${ }^{19}$ Esta práctica consistía en importar elementos de otros sectores productivos (en este caso el naval y el automovilístico respectivamente) y desarrollarlos de manera que pudieran pasar a formar parte de la industria de la construcción.

Concretamente John Dinkeloo, 20 empeñado en que el único camino de la edificación pasaba por acercarse a la precisión y perfección de industrias como la aeronáutica, fue el responsable de investigar cómo el acero cor-ten y las juntas de neopreno podian beneficiar la construcción de edificios y que así, esta disciplina ganase en innovación.

Pero en la fábrica para la Cummins en Darlington, KRJDA no se limitó exclusivamente a importar estos dos materiales a Inglaterra. Decidieron avanzar un paso más y convertir este edificio en el primero del mundo que utilizaba estos elementos tanto en el exterior como en el interior, ejecutando exactamente las mismas soluciones constructivas dentro y fuera. Este mecanismo sería utilizado con frecuencia en obras posteriores de KRJDA ya que favorece la ambigüedad entre el interior y el exterior que estos arquitectos buscaban constantemente. Esto también tiene una implicación positiva a futuro ya que, en caso de llevar a cabo las ampliaciones que se preveían (y que finalmente se acometieron en la década de los ochenta), la estructura que es exterior en el momento de la obra original, puede pasar a ser interior sin ninguna transformación.

Es interesante detenerse en la solución estructural de la fábrica, que está íntimamente relacionada con la distribución de las instalaciones, la necesidad de utilizar maquinaria de gran tamaño y la intención de mantener la misma solución en el interior y el exterior. $\mathrm{El}$ edificio se resuelve con un sistema de vigas, correas y elementos portantes de acero corten AS441 estable- 
Maqueta de estudio. En ella se aprecia la detallada y cuidada solución de la coronación del edificio emulando soluciones de la arquitectura clásica mediante la estructura, elemento expresivo del edificio. KRJDA.
Vista general de la fábrica. La horizontalidad es el rasgo formal más evidente del edificio, solo contrarrestado por la presencia de la chimenea, realizada también en el mismo material y colocada en el exterior del edificio, en continuidad con el bloque de servicios que separa la zona administrativa de la propiamente industrial. KRJDA.

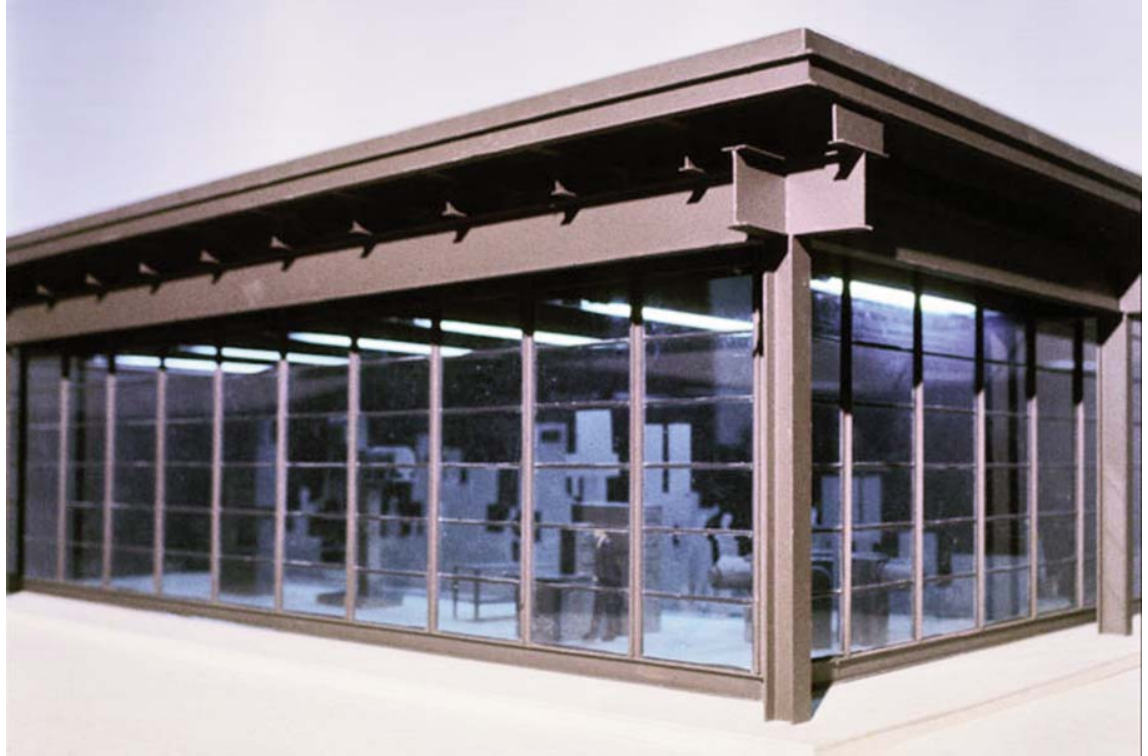

ciendo un módulo de 30 × 60 pies $^{21}$ que se extiende a todo el edificio y que se consideraba óptimo para el uso industrial.

El detalle que lo hace singular es que las correas de 12 pulgadas que se disponen en sentido transversal, se colocan sobre un pequeño apoyo (en este caso de 18 pulgadas) en vez de directamente sobre las vigas principales. Así, el espacio libre entre la viga y el plano inferior de cubierta aumenta hasta 30 pulgadas en sentido transversal, profundidad suficiente para alojar la instalaciones principales y de mayor tamaño (electricidad, aire, vapor...). En la dirección longitudinal, la profundidad utilizable es de 18 pulgadas (la altura del apoyo), espacio necesario para ubicar las ramificaciones de todas estas instalaciones.

Este cuidado tratamiento de los perfiles metálicos es evidente en el exterior y provoca la intensa expresividad estructural que define la imagen de este edificio. Se manifiesta una gramática sencilla en la que se podrian reconocer y revisar los elementos clásicos de los templos griegos aunque sin más detalles que los que ofrece el propio perfil metálico. Gracias a la colocación del apoyo entre las vigas y las correas, se pueden apreciar los tres elementos que conforman el entablamento: el arquitrabe estaria formado por las vigas principales, el friso estaría definido por las pequeñas ménsulas añadidas y los espacios vacíos entre ellas y la cornisa por la linea de cubierta que, en este caso y a diferencia de los templos, siempre es plana. Los pilares metálicos y exentos que rodean exteriormente este edificio culminan la imagen clásica de esta fábrica.

De nuevo, este edificio anuncia uno de los conceptos que sustentarán la obra de KRJDA en sus primeros veinte años de carrera. En obras como la torre Knights of Columbus o el New Haven Arena volverán a apostar por la expresión de sus elementos estructurales para enfatizar la imagen formal. Respecto a la fábrica de Walesboro, sin embargo, las diferencias son notables en este sentido, ya que en la planta americana la estructura permanece oculta y no es un rasgo que defina el proyecto. 
Planta de la fábrica Cummins en Darlington. KRJDA (Indicación de organización funcional por la autora)

Imagen interior de época. Aparecida en la revista Design en 1969.

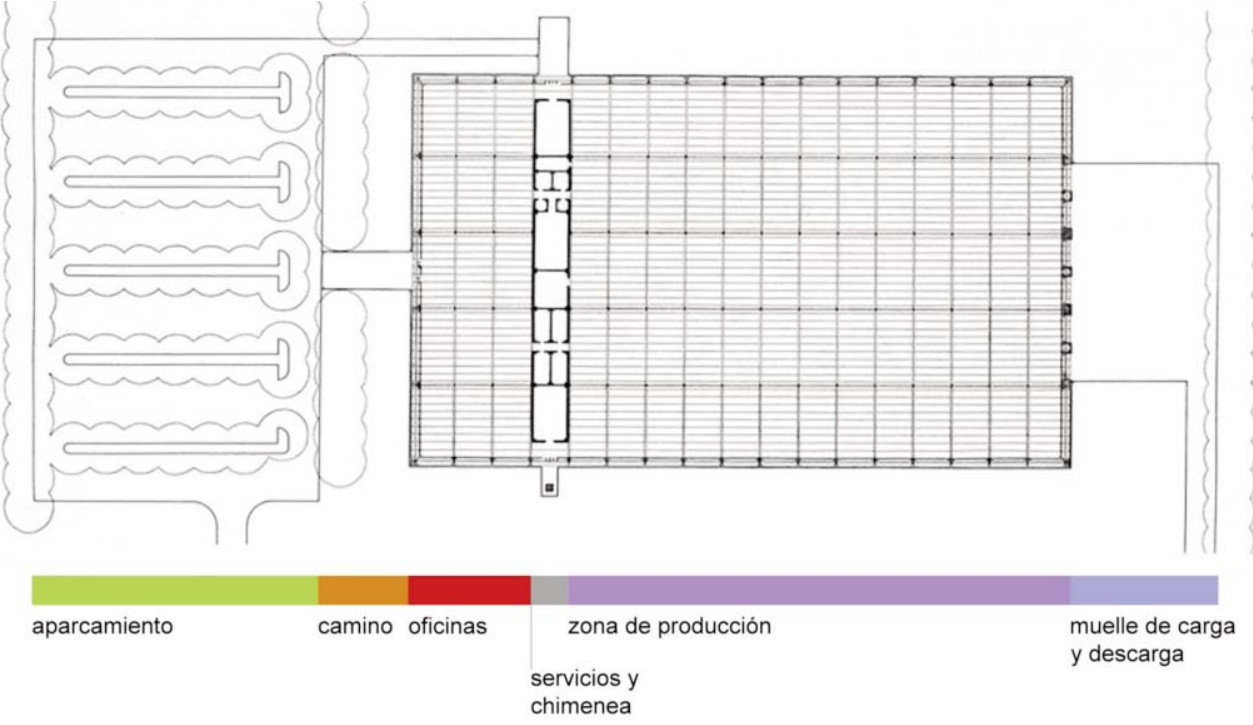

Un gesto que sí comparten las dos fábricas es una marcada horizontalidad. Se trata de dos edificios de gran dimensión en superficie y de una única planta en altura (en el caso de Walesboro, además, esta planta está semienterrada por lo que su presencia en el entorno es más sutil si cabe). En la fábrica de Darlington, la presencia de una chimenea introduce un claro contrapunto vertical.

En cuanto al funcionamiento interior, el edificio se rige por un principio de sencillez. La planta es un rectángulo de $510 \mathrm{x}$ 300 pies y alrededor de 22 pies de altura que se divide en dos zonas. En un extremo se ubican las oficinas y en el otro, la zona de maquinaria, separadas ambas por una crujia intermedia destinada a instalaciones y cuartos de almacenaje. Al utilizar estrictamente la misma solución constructiva a lo largo de toda la fábrica, no existe diferenciación espacial entre zonas, favoreciendo así la flexibilidad interior requerida. Ambas partes se tratan como zonas de trabajo abiertas que pueden variar de tamaño según las necesidades. Las oficinas y las zonas de producción se diseñaron para ser intercambiables, y no hay distinción de tipo de espacio entre trabajadores manuales y aquellos dedicados a labores administrativas. Esta decisión se entendía también como un beneficio de cara al futuro. En el caso de que la empresa decidiera mudarse y alquilar o vender el edificio, los futuros inquilinos tendrian un gran espacio libre para amoldar a sus necesidades.

En este edificio también se inician las primeras consideraciones que KRJDA hace sobre la democratización de los espacios de trabajo y la eliminación de jerarquías. Tanto operarios como directivos acceden por la misma entrada y entre ellos siempre existe contacto visual. Esto ocurre también en la planta de Walesboro de manera aún más evidente, ya que directamente propone un nuevo tipo de fábrica en cuanto a su organización funcional.

Aquí, desde el aparcamiento de coches exterior se accede al edificio a través de un camino con un cuidado tratamiento paisajístico; no hay que olvidar que Dan Kiley ${ }^{22}$ estuvo involucrado en este proyecto. La entrada principal dirige a los usuarios hacia las oficinas de dirección y los espacios de diseño de piezas, cercanas a la zona de gestión, departamento de personal y cantina. El proceso de producción comienza en el lado opuesto, con el muelle de carga y el almacén, desde donde se alcanza la zona de trabajo principal.

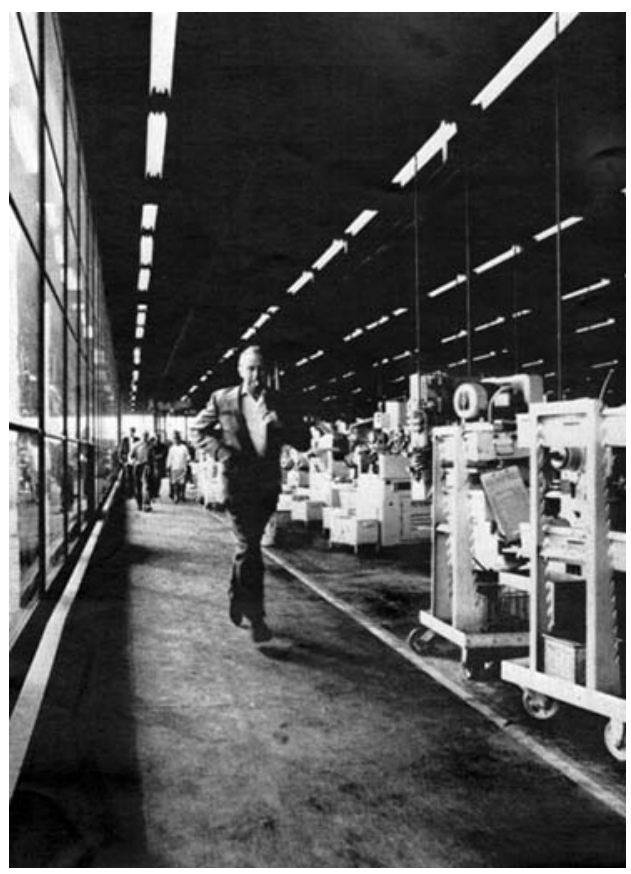




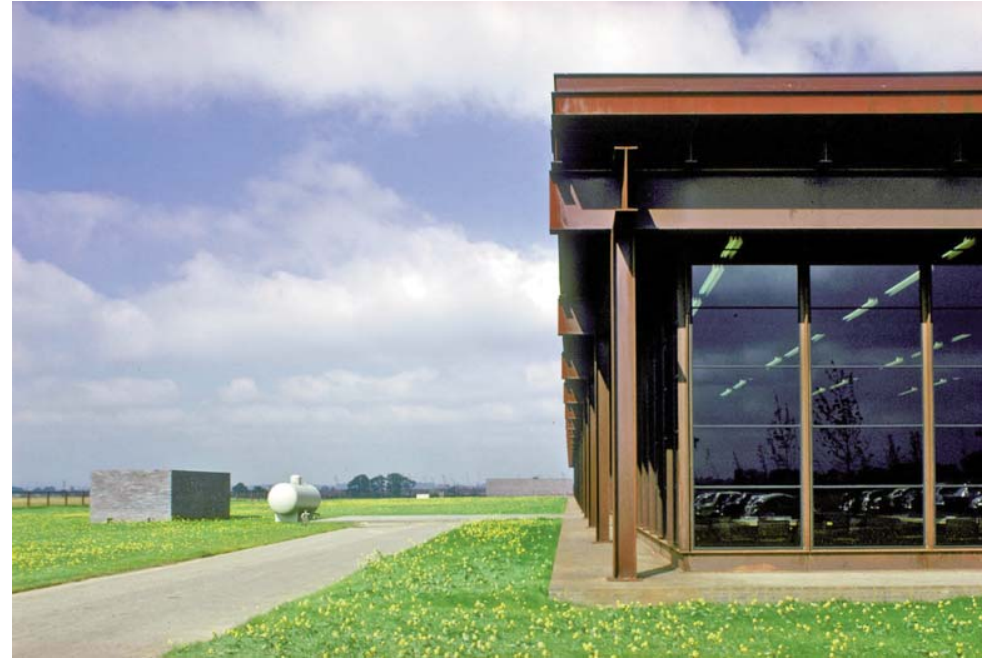

Detalle de esquina $y$ visión frontal de los vidrios del cerramiento. KRJDA.

Detalle de los vidrios especulares que reflejan la pradera del entorno. La unión entre vidrios en el despiece horizontal se ejecuta con juntas de neopreno. Imagen cedida por Roberto Osuna Redondo.
El espacio interior se concibe como un contenedor neutro, sin falsos techos e iluminado artificialmente con luminarias dispuestas en sentido longitudinal. Incluso la maquinaria se pintó en un color blanco suave para enfatizar la sensación de espacio limpio y tranquilo. ${ }^{23}$ Sólo destacan algunos paramentos verticales construidos en ladrillo vítreo de color azul. Quizá la única diferenciación entre la zona de oficinas y la de producción se encuentre en el acabado del suelo. Un pavimento blando de moqueta para los despachos y un pavimento duro, en continuidad con el patio trasero, para la parte industrial.

Todo este contenedor se envuelve en un cerramiento de vidrios tintados que arrancan en el suelo y acometen contra la parte inferior de las correas. Los vidrios se separan mediante montantes verticales metálicos siguiendo el ritmo de la modulación. En un segundo nivel de despiece horizontal los vidrios se enmarcan con juntas de neopreno negras. El cerramiento de vidrio gris-verdoso permite a los trabajadores las vistas hacia los campos que rodean al edificio pero evita la transparencia total hacia el interior, reflejando la pradera del entorno. Además, este tipo de vidrio, junto con el alero de cubierta, favorece la protección solar hacia el interior del edificio.

Por deseo expreso del cliente, y coincidiendo con la manera de trabajar de KRJDA, hubo un especial empeño por mantener la calidad en todas las fases de la obra. En este sentido, la presencia continuada de John Calvert durante la construcción resultó fundamental para la calidad y para la reducción de costes del proyecto así como la de Guy Martin, el encargado de la logística internacional por parte de la propiedad.
Durante las obras, el trabajo con el acero fue rápido y las soldaduras se ejecutaron correctamente por parte de los trabajadores locales, pero el vidrio tintado supuso más dificultades. Los paneles se fabricaron en la empresa Pilkington, la prestigiosa fábrica de vidrio, pero las juntas de neopreno que debían solucionar la estanqueidad aún no se habian utilizado como material de construcción en Inglaterra, y el primer intento no fue muy satisfactorio. Se tuvieron que rechazar el 75 por ciento de las juntas que se fabricaron allí por ser insuficientemente precisas. La solución que se consideró más rápida y económica fue importar directamente de Estados Unidos las juntas que se colocarian en el interior.

Finalmente, la fábrica se terminó de manera satisfactoria para todos los agentes ${ }^{24}$ y por un precio que en esos años se equiparaba al de otros edificios sin la calidad técnica y las singularidades de diseño presentes en este pabellón.

La repercusión de este proyecto en los medios de difusión arquitectónica fue importante. Apareció publicada por primera vez en la revista Architectural Forum en octubre de 1966,25 sólo un mes después de la formación oficial de Kevin Roche John Dinkeloo and Associates como estudio. Por lo que se considera su primer edificio construido.

En julio de 1967, la revista inglesa Architectural Review publicaba un texto titulado "Made in Mid-Atlantic"26 en el que elegía

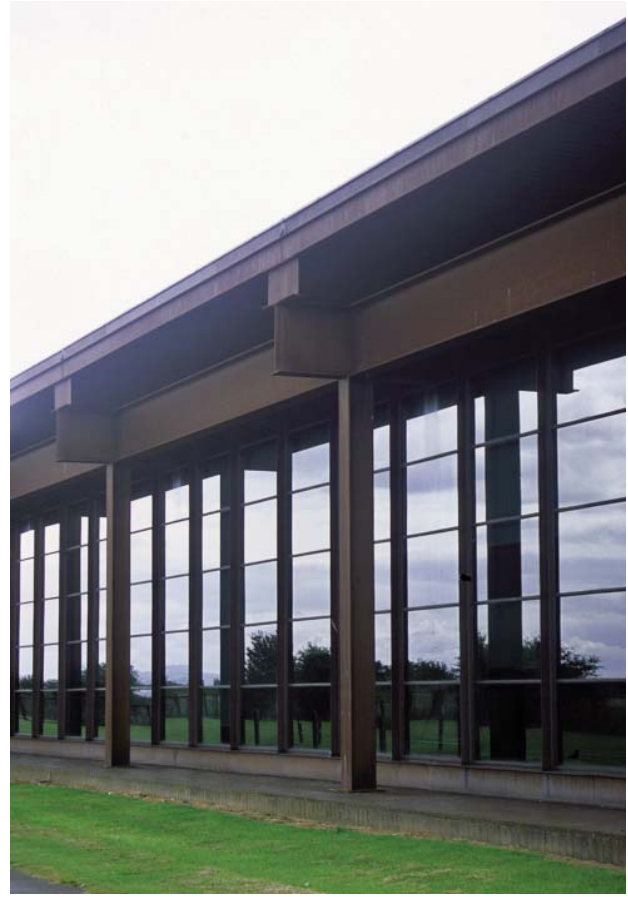




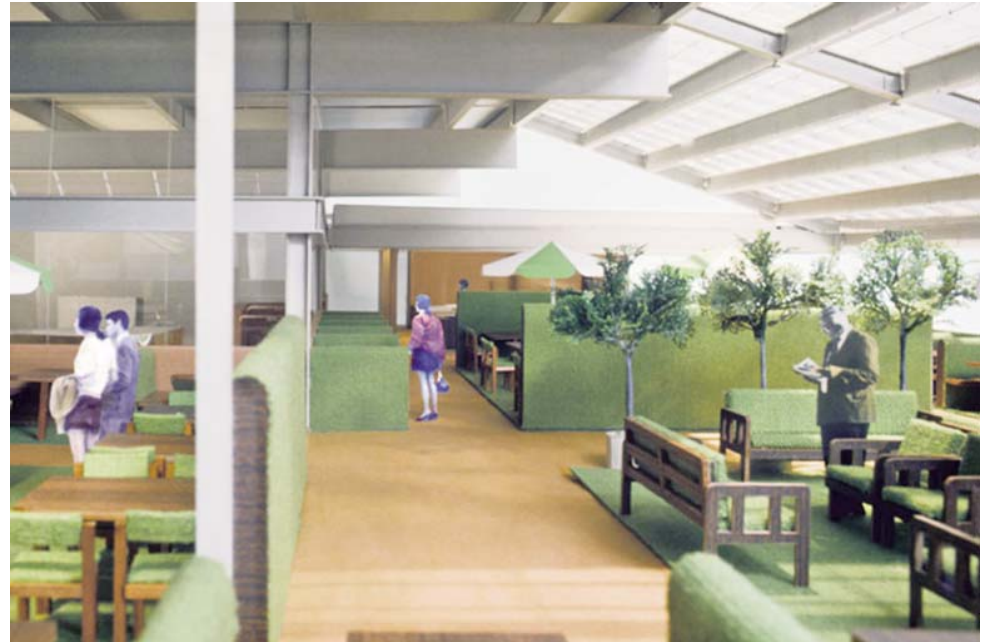

Montaje del interior del espacio de oficinas diseñado en la fábrica de Walesboro por KRJDA. (Ref. web 3)

cuatro proyectos industriales que mostrasen alguna relación entre Estados Unidos y Reino Unido. El caso de la fábrica de Darlington era claro: edificio en suelo inglés, de arquitectos americanos ${ }^{27}$ y para una empresa estadounidense. Los otros ejemplos que aparecian en el artículo fueron Team 4, con su fábrica para Reliance Controls en Swindon, Marcel Breuer con la fábrica Torrington y, por último, Max Gordon, con el edificio para Commercial Plastics en Cramlington. En este texto valoraban que todas las fábricas trabajaban sobre la idea de gran contenedor lineal, con espacios interiores amplios y flexibles. Destacaban la fábrica para la Cummins Engine Company como "quizá, el edificio construido en Inglaterra concebido de manera más profesional desde la Segunda Guerra Mundial".28 Un mes después, Architectural Design también presentaba el edificio al público inglés, esta vez con un artículo de menor extensión y más descriptivo.

A partir de 1968 surgen algunos recopilatorios $^{29}$ del trabajo de KRJDA que muestran el interés que despertaban estos arquitectos aunque su obra construida era aún escasa. El edificio de Darlington aparecía en todos estos artículos y sus valores más destacados volvian a ser la expresividad estructural y el uso de nuevas soluciones constructivas.

\section{Sub-assembly plant Cummins Engine Company, Walesboro (1975)}

Pocos años después, la misma empresa volvió a confiar en Kevin Roche John Dinkeloo and Associates para diseñar una gran planta manufacturera en Walesboro, un lugar rural al suroeste de Columbus.

J. Irwin Miller, cliente de nuevo, buscaba arquitectos que investigasen en profundi- dad el programa y las necesidades funcionales con el objetivo de conseguir una solución óptima. Como cliente, Miller apreciaba la sensibilidad de Kevin Roche para captar esos requisitos sin prejuicios que tuvieran que ver con la forma o un estilo concreto.

De hecho, es uno de los predicamentos de Kevin Roche, quien afirma continuamente en sus entrevistas que la verdadera razón para que un edificio exista es su interior, el lugar donde se produce la acción para la que se ha destinado. En este sentido, se involucra al máximo en que la estancia de las personas en el interior del edificio, sea lo más agradable posible, y especialmente en aquellos espacios dedicados al trabajo ya que es donde pasan la mayoría del día. En Walesboro se hace patente esta inquietud y el edificio se inicia pensando el interior, cómo relacionar a las personas que trabajan en él y cómo ofrecerles un entorno agradable.

Aunque la vista del bosque exterior es constante desde el interior, se pretende que también se pueda disfrutar del interior del propio edificio. En la obra de Roche y Dinkeloo es muy común que los usuarios sean conscientes en cualquier momento de la experiencia del edificio que están ocupando. El ejemplo más evidente es la Fundación Ford, pero también ocurre en esta fábrica de Columbus y de alguna manera también pasaba en Darlington. En la planta inglesa el mecanismo para conseguir trasladar esta presencia al interior era el uso de las mismas soluciones constructivas dentro y fuera del edificio. En la fábrica americana, el interior se disfruta gracias, entre otras cosas, a la decoración de oficinas, que evocan un patio soleado, con mesas de picnic de madera, bancos, sombrillas..

Pero no es únicamente la decoración relajada lo que convierte la fábrica en un espacio de trabajo sugerente sino la propia organización funcional de la planta, que supuso un cambio en la manera de entender los espacios industriales y las relaciones que debian establecerse entre los distintos trabajadores que ocupan una fábrica. Las innovaciones funcionales propuestas por Roche y Dinkeloo tuvieron un rápido reconocimiento $\mathrm{y}$, a principios de los años setenta, sin estar finalizado todavía, el edificio fue proclamado como un prototipo a seguir para futuros edificios industriales. ${ }^{30}$

La primera cuestión a tener en cuenta en este proyecto era el tamaño de la fábrica, 570.000 pies cuadrados que prácticamen- 
Imagen aérea de la planta de la empresa Cummins en

Walesboro. Para la ubicación del edificio se eligió una zona despoblada de árboles y se situó el aparcamiento en la extensa cubierta de 570.000 pies cuadrados para no asfaltar el terreno de alrededor ni encarecer el proyecto mediante excavaciones innecesarias. KRJDA.
Detalle de la solución constructiva del edificio. En este caso, y al contrario que en Darlington, la estructura metálica solo se aprecia desde el interior. La presencia exterior del edificio es muy sutil, jugando con la idea de "no-edi ficio". El conjunto se entierra en dos niveles distintos y se utilizan paños de vidrio para camuflar el edificio en su entorno. El aparcamiento de cubierta se remata con un peto retranqueado que imposibili ta la visión de los coches desde el exterior. KRJDA.

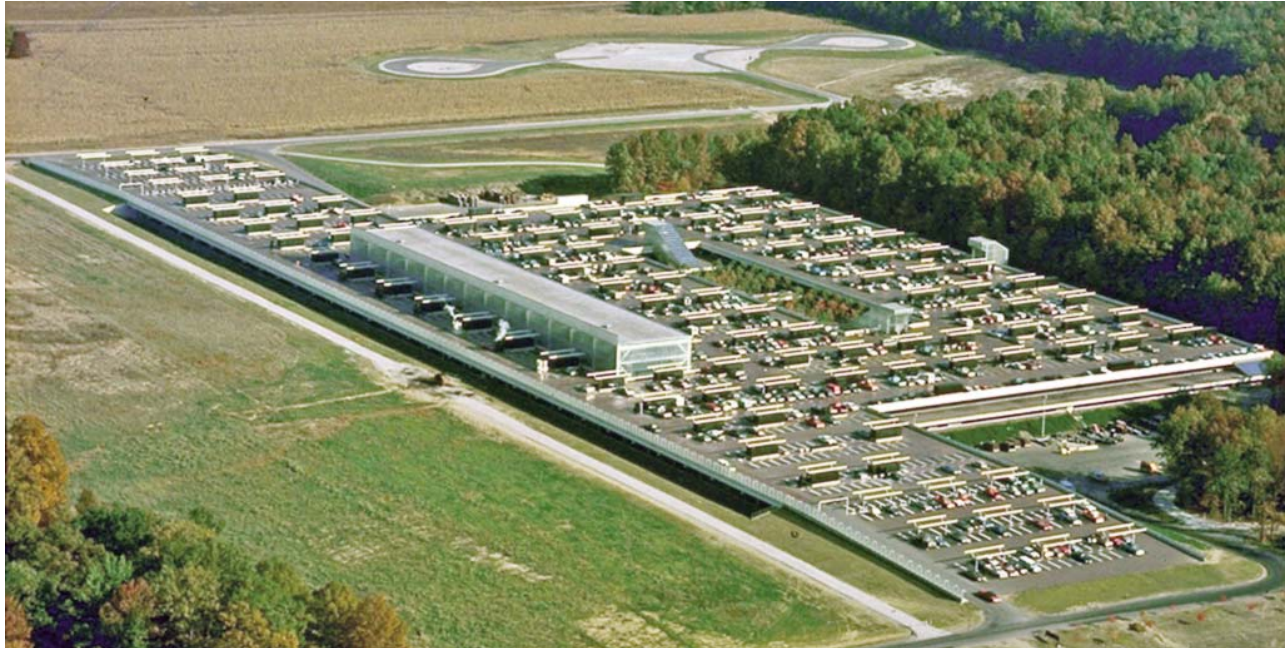

te cuadruplicaban los poco más de 150.000 pies cuadrados de la planta de Darlington. Obviamente, la capacidad era mucho mayor por lo que el reto fundamental era gestionar el flujo de las más de dos mil personas que la ocuparian. Además, debido a la localización de la fábrica se asumia que éstas tendrían que acceder al complejo en coche. De hecho, el primer requerimiento funcional fue un aparcamiento de 1400 plazas.

La interacción entre el vehículo y la arquitectura es un tema ampliamente abordado por Roche y Dinkeloo desde el inicio de su trayectoria. El automóvil privado se había impuesto como un elemento primordial de la vida americana desde unas décadas atrás. Muchas empresas, la Cummins entre ellas, se habian desplazado desde las ciudades a entornos rurales en busca de suelo económico y mejores perspectivas para su identidad corporativa al alejarse de las restricciones normativas urbanas. Esto obligaba a los trabajadores a usar el vehículo diariamente, convirtiéndose prácticamente en la prolongación de su vida doméstica.
La conservación del entorno natural era una obsesión para KRJDA, que no consentían la tala indiscriminada de árboles para asfaltar el terreno alrededor del edificio obligando a los usuarios a largas caminatas hasta llegar a su puesto de trabajo. Investigaron las posibilidades de reducir las distancias a recorrer desde el aparcamiento hasta el asiento de los trabajadores $\mathrm{y}$, en el caso de Walesboro, decidieron aprovechar la extensa cubierta del edificio para ubicar las 1400 plazas solicitadas. La vista aérea del edificio, plagada de coches, se convirtió rápidamente en el rasgo distintivo de este proyecto.

La cubierta estaba calculada con una estructura de acero reforzada para alojar las grúas y la maquinaria que se utilizaba en la fábrica así como para las cargas de nieve habituales en la zona. Los ingenieros Pfisterer Tor Associates, colaboradores habituales de KRJDA, consideraron que estos cálculos permitían también añadir la carga aportada por los automóviles.

Esta cubierta estaba ligeramente elevada sobre la cota del terreno y, además, el

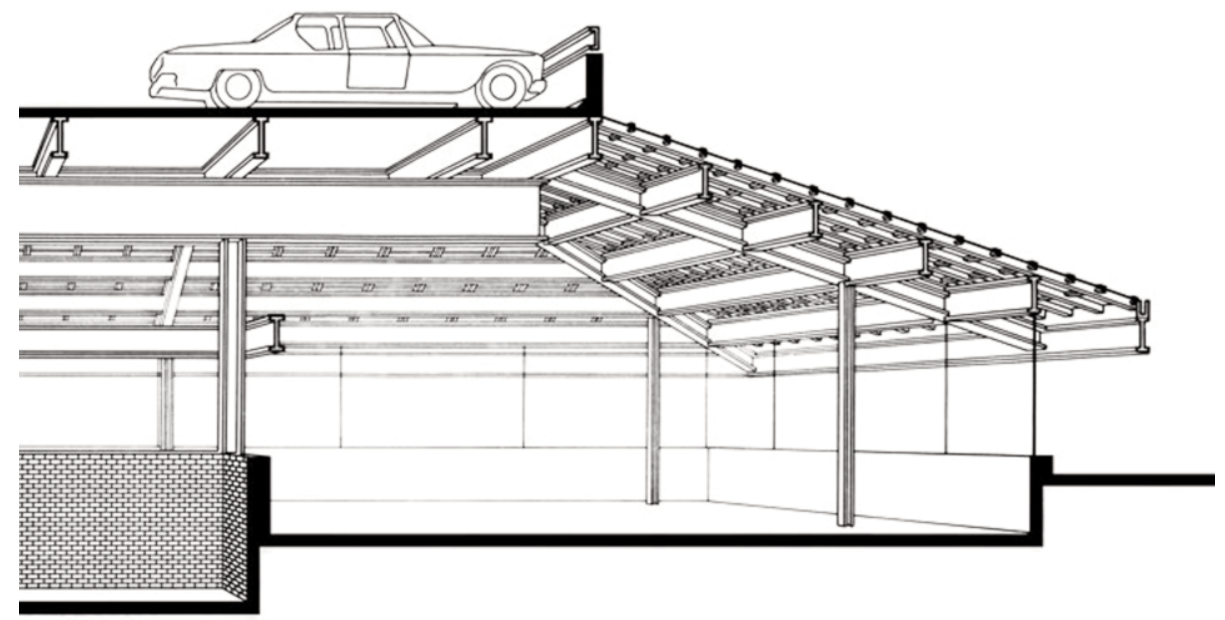


Patio central ajardinado con dos de los accesos al interior de la fábrica. Al fondo, pérgolas del aparcamiento. KRJDA.

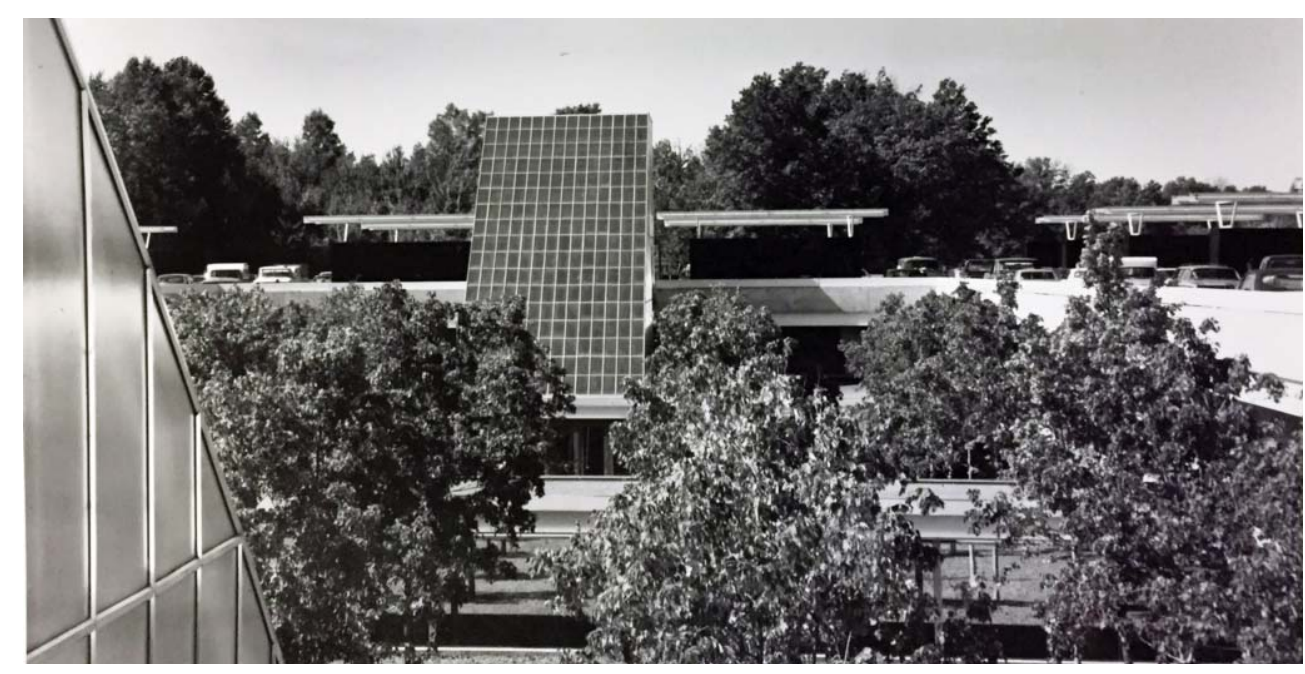

aparcamiento se esconde tras un pequeño peto retranqueado desde la línea de cornisa por lo que la vista de los automóviles desde el exterior es imposible.

Una vez aparcado el coche, cada trabajador accede al edificio por una de las entradas dispuestas en fachada, aquélla que le deje más cerca de su zona de trabajo. Estas piezas de acceso se construyeron en vidrio, como el resto del cerramiento del edificio y se adaptaban a la geometría de las escaleras que contenían mediante planos inclinados.

Merece la pena recordar que KRJDA también hizo aportaciones al desarrollo de nuevos vidrios para la industria de la construcción. John Dinkeloo trabajó con fabricantes sobre diferentes tratamientos para los vidrios que luego aplicó en sus edificios. Son especialmente recordados por el desarrollo del vidrio especular para las fachadas de los edificios pero también ensayaron distintas formas de protección solar o incluso soluciones que, estéticamente, resolvieran las necesidades del proyecto en cuestión. Además, aprovecharon las ventajas del vidrio para jugar con geo- metrias nuevas, fundamentalmente con planos inclinados como en el United Nations Plaza o la galería del Irwin Bank.

Ya en el interior de la fábrica, el espacio está organizado por un patio central en torno al que se ubica la zona de producción. La singularidad del proyecto recae en la relación que se establece entre la parte dedicada a oficinas y la destinada a maquinaria. Roche y Dinkeloo se alejan de soluciones convencionales en las que las zonas administrativas se colocan en plantas distintas o alejadas de la fábrica en sí y ubican las oficinas en los tres lados perimetrales del edificio que rodean el espacio industrial. Así, la relación visual entre todos los trabajadores es continua, independientemente de su tipo de trabajo o jerarquía. Esta conexión entre empleados ya se había iniciado en Darlington, aunque de manera más sutil, y es un exponente del pensamiento de KRJDA en cuanto a los edificios corporativos e industriales.

Las oficinas y la fábrica se miran constantemente a través de las mamparas de vidrio que las separan, permitiendo también el paso de luz y vistas lejanas a través
Dibujo de proyecto de KRJDA donde se aprecia la diferencia de cota entre el centro del edificio y el perimetro asi como las entradas de luz natural y la relación del interior con el paisaje exterior. (Ref. web 3)

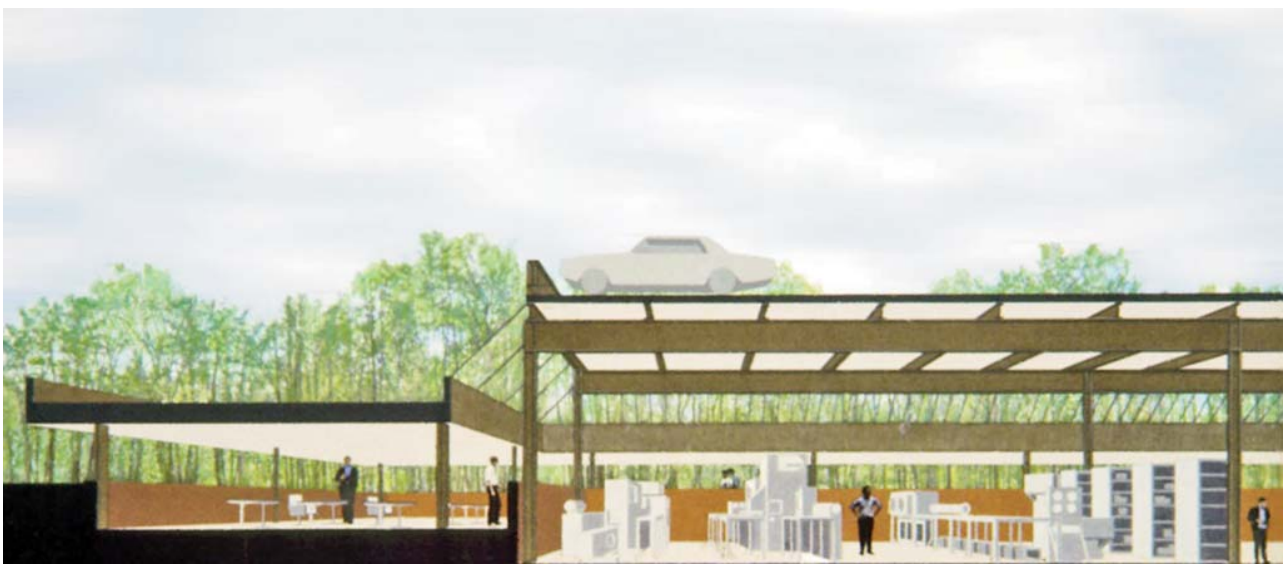




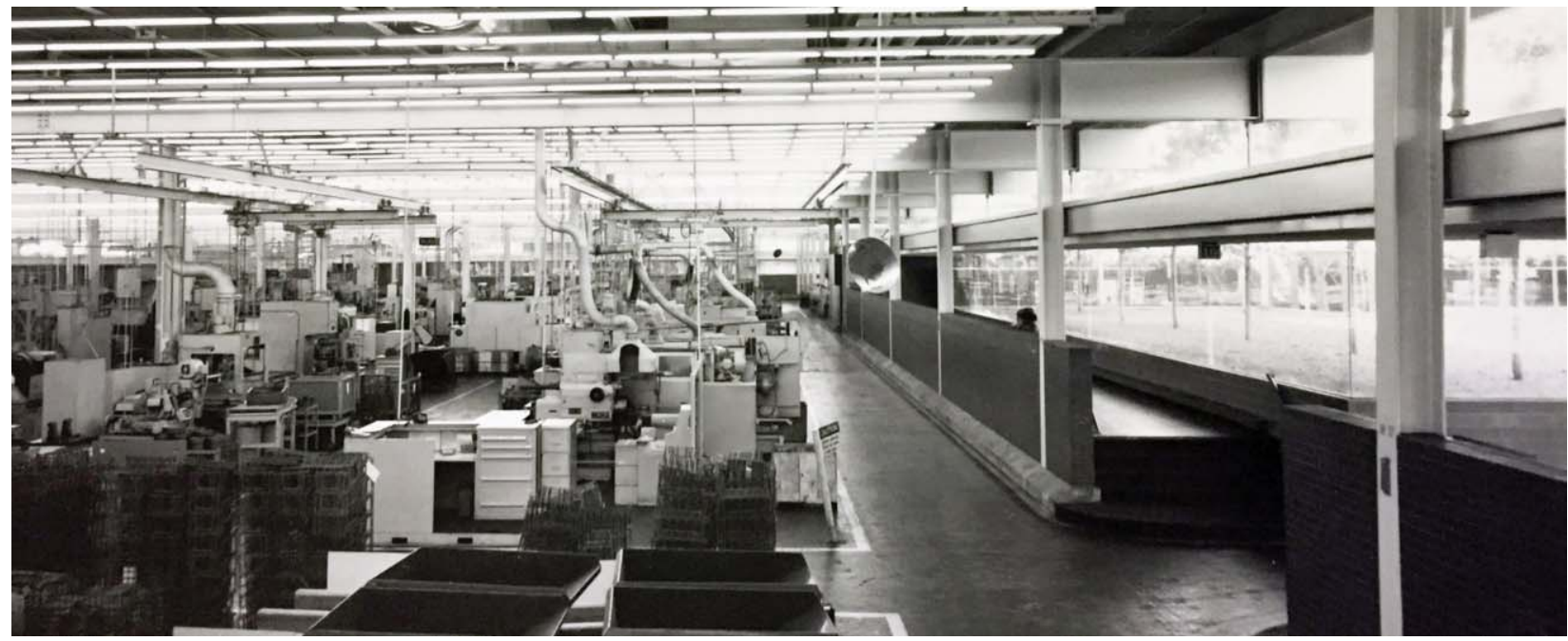

Imagen de época del interior de la fábrica con la maquinaria ya instalada. A la derecha, el patio central que conecta con la zona de trabajo. KRJDA.

Planta de la fábrica de Walesboro. (Organización funcional por la autora sobre dibujo de KRJDA.)

Sección transversal por el patio y la zona de almacenaje a doble altura. KRJDA. del edificio. Las oficinas se entierran solamente tres pies bajo tierra mientras que el espacio central se hunde otros tres pies para cumplir con las necesidades de la maquinaria, así como para conseguir una proporción adecuada para un espacio de tan grandes dimensiones.

La percepción visual del arbolado exterior es permanente desde cualquier punto. Para los trabajadores de oficina ésta se produce directamente a la altura de los ojos y para los empleados de la fábrica se juega con una vista más cenital. Entre la cubierta del espacio central y la del perímetro, se produce una fisura que se cierra con planos inclinados de vidrio que iluminan naturalmente el interior de la fábrica a la vez que introducen el paisaje vegetal dentro del edificio.

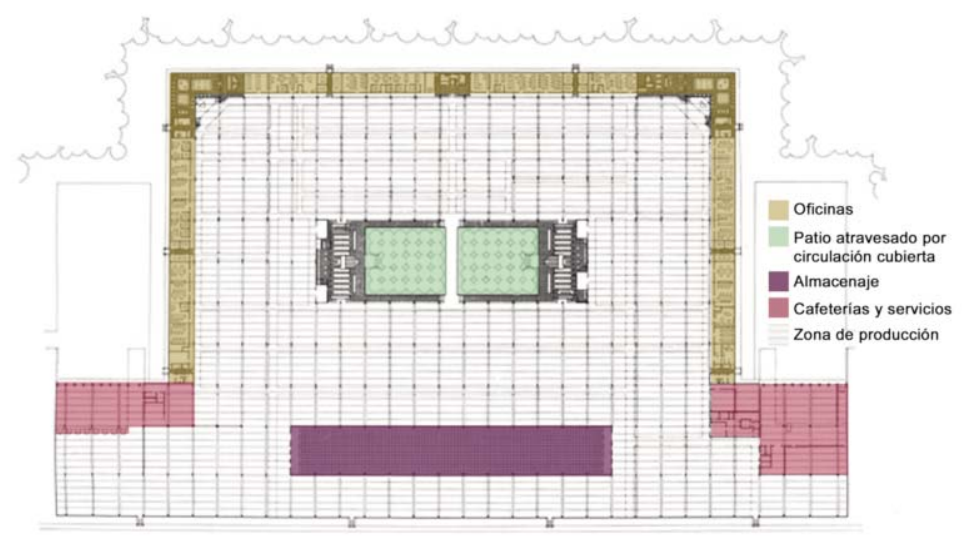

"Esta planta permite que cada trabajador tenga vistas al campo, y al poner el aparcamiento en el tejado, trae a cada trabajador a unos pocos pasos de su puesto, eliminando la necesidad de acres de asfalto para aparcamiento". 31

Las palabras de Miller no podian estar más en consonancia con las ideas de KRJDA para este proyecto. Tanto la propiedad como los arquitectos asumieron la responsabilidad de crear una fábrica alejada de convencionalismos.

En esta ocasión, el contenedor sencillo y directo que funcionaba en Darlington adquirió mayor complejidad espacial y se introdujeron nuevos elementos. Uno de los motivos es la mayor extensión del edificio de Walesboro, pero también influyó la concepción de la fábrica americana como un edificio acabado, sin la posibilidad de ampliaciones que se requería en la planta de Inglaterra.

Para completar la organización funcional de la planta, el edificio se cierra con una banda dedicada a almacenamiento y logística. Se trata de un cuerpo que ocupa trece módulos estructurales y que se separa del resto de la fábrica mediante muros de hormigón. Recibe luz natural desde el techo ya que es el espacio de mayor altura de la nave, el prisma que sobresale discretamente de la cubierta sin condicionar en ningún momento la horizontalidad que presenta el edificio.

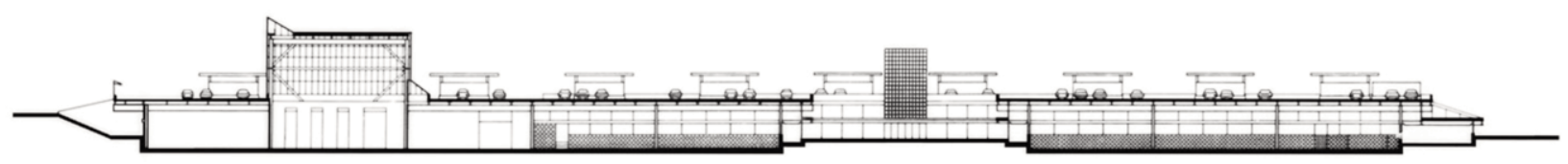




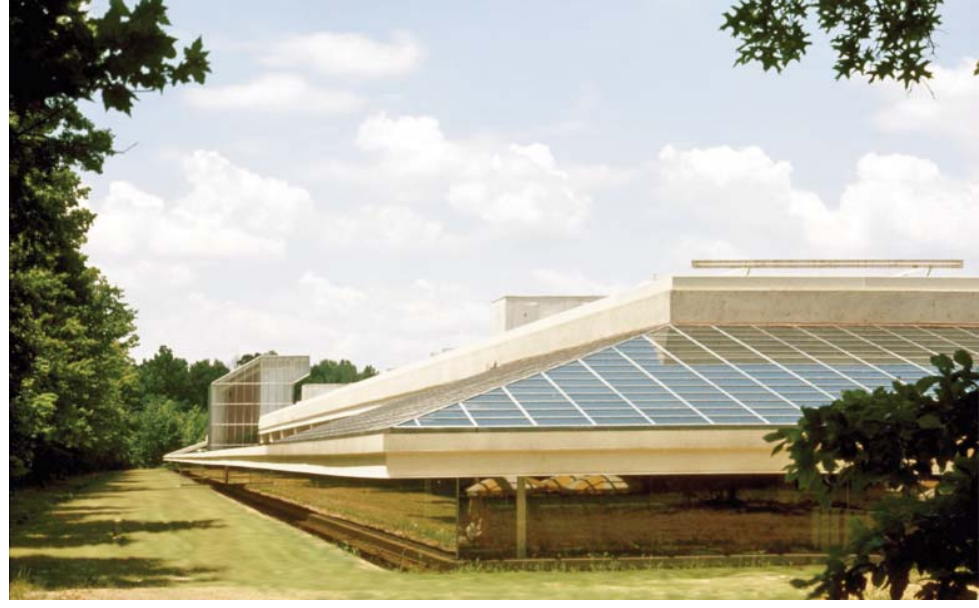

Detalle de esquina del edificio. El peto del aparcamiento $y$ la linea de remate de cubierta enfatizan la sensación de horizontalidad del edificio. Sólo pequeñas piezas de vidrio interrumpen este carácter en determinados puntos.

Imagen general de la fábrica. Los cerramientos de vidrio se valoran también por la iluminación artificial del interior. KRJDA.

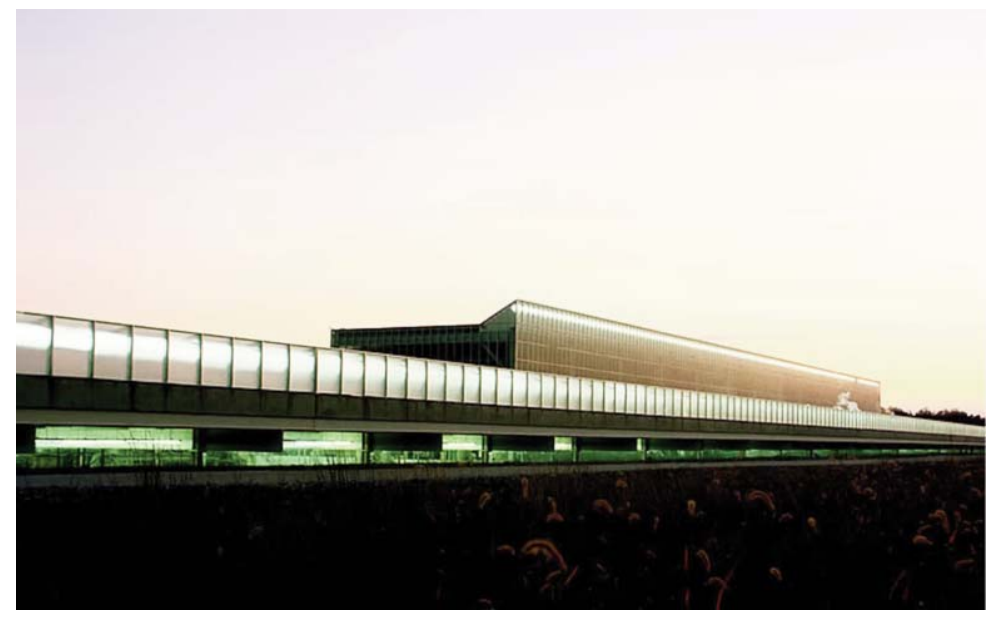
en primer lugar por se inserta en el entorno existente. Se trabaja sobre la idea de un "no edificio" que, al estar semienterrado, emerge ligeramente del terreno. Aunque la proporción ya enfatiza la horizontalidad del proyecto, las decisiones constructivas también apoyan esta imagen. El edificio mantiene líneas horizontales definidas por remates constructivos con el objetivo de reforzar este carácter.

Además, los arquitectos aplicaron distintos recursos formales para el cerramiento de vidrio que otorgan riqueza al resultado formal final. Utilizaron geometrias inclinadas entre las lineas horizontales para que los planos se percibieran lo menos posible en favor de las líneas. Recurrieron al vidrio plano, sin juntas y retranqueado, a fin de que el edificio pareciese que flotaba sobre el terreno. También aprovecharon la iluminación artificial interior para producir efectos sobre el cerramiento de vidrio exterior y, por último, apostaron por la capacidad del vidrio para dar lugar a nuevas geometrias con el objetivo de crear pequeñas piezas escultóricas en las que alojar las comunicaciones verticales.

El propio Kevin Roche manifestaba recientemente que se sentía orgulloso del resultado de este proyecto y lo definía como el "mejor edificio industrial que hemos realizado en KRJDA". 32

Al igual que la fábrica para la Cummins en Darlington, este edificio también tuvo repercusión en los medios especializados. En 1975 la revista Architectural Record la describía en su artículo "Productive elegance for industry" como "uno de los mejores lugares para trabajar -y para pasar una buena parte de la vida productiva. Y además, es una contribución a su entorno." 33 De estas palabras se deduce que el objetivo de los arquitectos de crear un lugar amable para los empleados estaba más que conseguido.

Un año antes, el monográfico ${ }^{34}$ que la revista Architectural Forum editó sobre el equipo dedicaba uno de los artículos que lo componen a la fábrica de Walesboro. En sus cuatro páginas destacaba que la fascinación por el vidrio y el empeño en resolver las demandas funcionales de la fábrica traían a la memoria a los primeros modernos: la fábrica de turbinas AEG de Behrens, la fábrica Fagus de Gropius y Meyer, la Werkbund, por no mencionar las de Albert Kahn en Estados Unidos u Owen Williams en Inglaterra. ${ }^{35}$ Pero también enfatizaba que KRJDA todavía avanzaban más que estos antepasados en cuanto al concepto de desmaterialización de la fábrica.

Antes incluso de estar finalizado el proyecto, también se pudieron ver imágenes de la planta de Walesboro en publicaciones como Horizon, ${ }^{36}$ pero para su difusión fue más importante la inclusión de una maqueta de la fábrica en la exposición del Museum of Modern Art de Nueva York entre octubre de 1970 y enero de 1971: "Work in Progress: Architecture by Philip Johnson, Kevin Roche, Paul Rudolph". Al Arthur Drexler, comisario de la exposición, le interesaba especialmente este proyecto por su utilización del vidrio, tanto en paredes como en cerramientos cenitales, pero sobre todo por la respetuosa relación que se establece entre la arquitectura y el paisaje. 

fábrica se camufla en el entorno boscoso en el que se inserta. Imagen cedida por Maria Teresa Valcarce Labrador.
Durante el día, la
Imagen actual de la fábrica de Darlington con la chimenea en primer plano. Imagen cedida por Roberto Osuna Redondo.

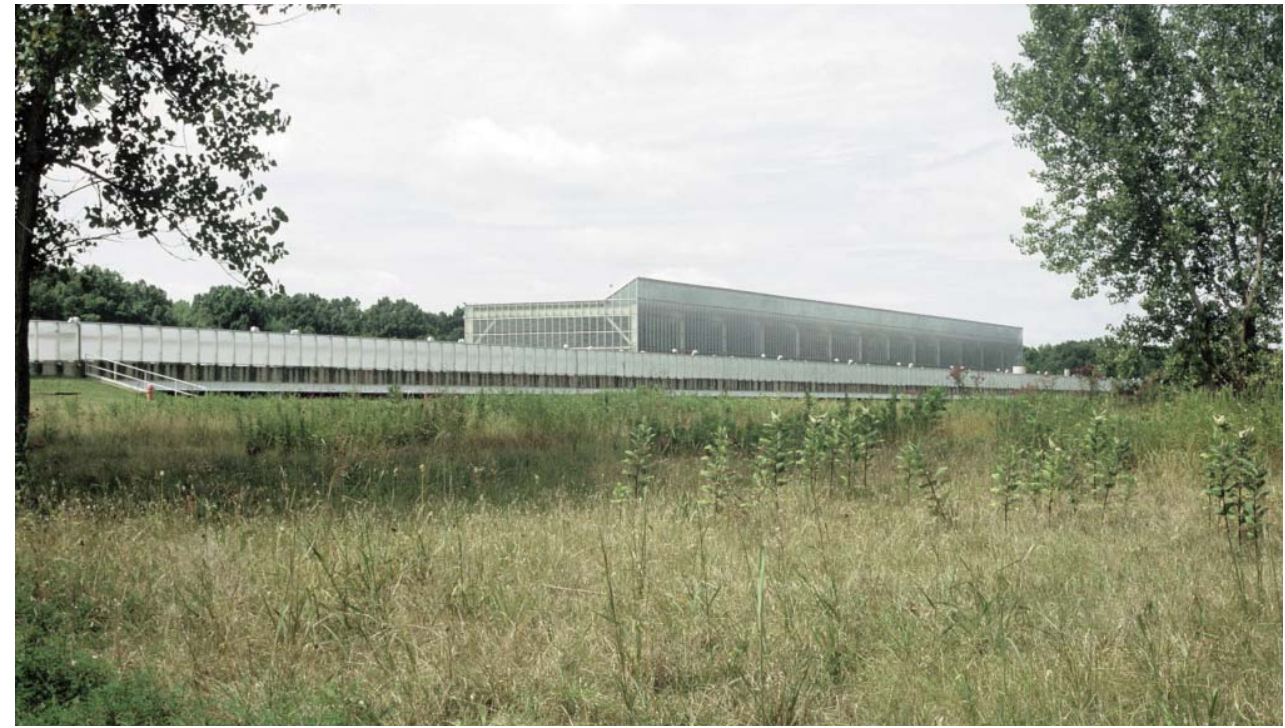

\section{Dos iconos del Patrimonio Industrial del} s. XX

Estos dos edificios no sólo han resistido el paso del tiempo sino que se han convertido en dos obras fundamentales de la arquitectura industrial del pasado siglo.

La planta de Darlington celebra su cincuenta aniversario este año y sigue considerándose uno de los mejores edificios industriales de Inglaterra, a pesar de haber atravesado diferentes circunstancias en estas décadas. ${ }^{37}$ En 1992, la revista The Architects' Journal iniciaba con este proyecto una serie de revisiones de edificios de los años sesenta y gracias a este texto se pueden conocer detalles del paso del tiempo por esta fábrica. ${ }^{38}$

Según el artículo, la modificación más reseñable ocurrió en 1980, cuando se amplió en 60 pies hacia el sur bajo la dirección de James Cubitt \& Partners, añadiendo módulos tal y como se había previsto durante la fase de proyecto. La

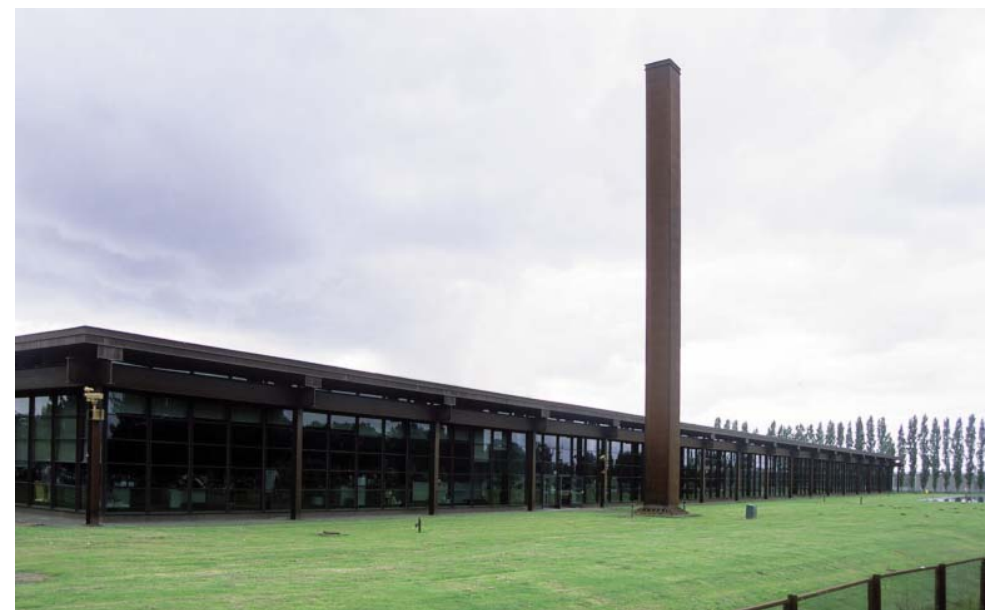

operación fue satisfactoria y las nuevas vigas se soldaron a la estructura existente sin afectar el funcionamiento de la planta. La ejecución fue tan cuidadosa que, según el autor, la intervención prácticamente pasa desapercibida. Sólo pequeños detalles delatan esta ampliación, como las placas base de la fila de pilares que ahora pasan a ser interiores cuando antes habian estado expuestas al exterior o la asimetría que se origina al quedar la entrada principal descentrada con la adición de un módulo.

Años después, debido a estrategias empresariales, la empresa tuvo que deshacerse de una de sus dos plantas en Darlington y decidieron desprenderse del edificio de KRJDA. Alan Richardson, encargado de las instalaciones de Cummins UK, se lamentaba de "tener que abandonar un edificio que había funcionado con gran éxito y tenía un coste de mantenimiento muy bajo". 39

En marzo de 1992, cuando se publicó el artículo en The Architects' Journal, la fábrica llevaba 18 meses vacía, con el consiguiente deterioro que sufren los edificios desocupados. El autor afirmaba en este texto que se habian cambiado algunos acabados interiores pero que, aun así, seguía mostrando un aspecto excelente a excepción de las instalaciones, ya obsoletas.

El dos de octubre de 1992, la revista británica Building Design publicó un artículo con tres edificios industriales posteriores a la Segunda Guerra Mundial elegidos por Patrimonio para su conservación. ${ }^{40}$ Además del edificio de la Cummins, se consideró una azucarera en Liverpool (195557), de los arquitectos Tate \& Lyle's Engi- 


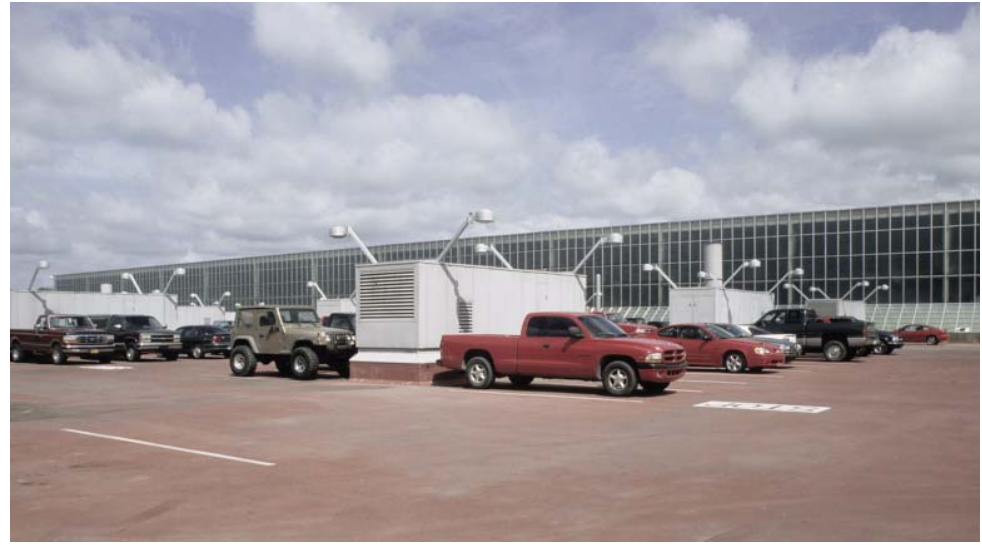

Imagen actual del aparcamiento de la fábrica de Walesboro con la pieza de alma cenaje a doble altura al fondo. Imagen cedida por María Teresa Valcarce Labrador.

neering Department y las oficinas, almacenes y destilerias Gilbey en Harlow (196263), de Peter Falconer \& Associates. La noticia debía estar escrita anteriormente a la publicación del artículo ya que la fábrica Cummins en Darlington se encuentra protegida desde el día 23 de septiembre de 1992 con Grado II*. Esto significa que es un "edificio particularmente importante de algo más que especial interés." Quizá por no tener la categoría de Grado I volvió a estar en peligro según recogía la revista Architectural Review en 1998.41

La fábrica de Walesboro también sufrió modificaciones y añadidos, pero en este caso no estaban previstos. David Powrie, arquitecto asociado de KRJDA, escribió el siete de octubre de 1985 a Susan Anderson, de la Cummins Engine Company, tras una visita a Columbus para expresar su malestar por las actuaciones llevadas a cabo, "que ya estaban afectando el carácter del edificio". ${ }^{42}$ Para solucionar estas circunstancias, el estudio le propuso a la propiedad un plan de actuaciones a seguir con el objetivo de adecuar la fábrica a las necesidades de la empresa sin desvirtuar la esencia del proyecto original.

En 1992 la empresa decidió emprender una remodelación y volvieron a contratar a Kevin Roche (John Dinkeloo había fallecido once años antes) para reconvertir la planta en un centro más dedicado a la ingeniería y la investigación. En palabras del propio Miller justo ese mismo año, la fábrica "ahora acoge las más avanzadas técnicas manufactureras que se conocen, y el edificio de 25 años ha requerido sorprendentemente muy pocas modificaciones».43 La planta de Walesboro sigue en pleno funcionamiento y continúa perteneciendo a la misma empresa.

En la actualidad, esta fábrica está reconocida como una de las obras más importantes del patrimonio industrial de los EEUU y forma parte de los atractivos de la ciudad de Columbus, Indiana, admitiendo incluso visitas para dar a conocer los valores del edificio.

En conclusión, se considera que los dos edificios analizados en el texto merecen ocupar un lugar destacado dentro del Patrimonio Industrial del siglo XX. En ambos casos, el resultado final se tradujo en obras de gran calidad arquitectónica que han sido valoradas por las instituciones competentes al concederles distintos grados de protección. Pero además, es necesario reconocer la labor de investigación previa que acompañó a ambos proyectos y que logró abrir nuevos caminos para mejorar la arquitectura industrial de los años posteriores.

\section{Notas}

1. En 1954, J. Irwin Miller crea la Cummins Foundation con el objetivo de fomentar la excelencia en la arquitectura de Columbus, Indiana, sede principal de la compañia. La fundación se comprometía a recomendar arquitectos de prestigio y a abonar los honorarios de los mismos para proyectos públicos de interés para la comunidad (fundamentalmente edificios religiosos y escolares). Asi, la ciudad cuenta con obras de Eliel y Eero Saarinen, Edward Larrabee Barnes, Robert Venturi o I.M Pei, entre otros.

2. Recuérdese que John Dinkeloo falleció la noche del 15 de junio de 1981 de un ataque al corazón, a la edad de 63 años (Glueck 1981).

3. Este término lo acuñó la crítica al trabajo de Eero Saarinen en repetidas ocasiones y, posteriormente, les sirvió también para comparar a maestro con sus herederos. Son muchas las publicaciones donde se puede encontrar, un ejemplo sería: Dark steel pavilion designed for export. 1966. Architectural Review 827: 2.

4. Traducción hecha por la autora del término "stylistic inconsistency," repetidamente utilizado por la crítica para referirse al trabajo de KRJDA. Se trata de una consecuencia derivada de la búsqueda del "style for the job" documentado en el punto anterior. Un ejemplo de este término se puede encontrar en: Wagner, Walter F. 1968 Fresh forms and new directions from a special kind of problem solving. Architectural Record 5: 145.

5. Ref. web 1: Modernism in Bartholomew County, Indiana, from 1942. United States Department of Interior, National Park Service. https://www.nps.gov/nhl/learn/specialstudies/ModernismBartholomewIN.pdf (visitado 26 de febrero de 2014) p: 4

6. J. Irwin Miller siempre mostró interés por el diseño y en la Universidad de Yale, donde se graduó, asistió a algunos cursos sobre arquitectura. (Ref. web 2.)

7. Inicialmente llamada Tabernacle Christian Church

8. En este breve texto, J. Irwin Miller expone su opinión como cliente sobre el trabajo de Roche y Dinkeloo. Futagawa 1975.

9. "Kevin demonstrated two characteristics, which still mark his work: his willingness to change his design when it clearly does not please a client, and as well his unwillingness to agree to any design that does not please him." Carta de J. Irwin Miller al AIA fechada a 2 de octubre de 1992 (Washington D.C: American Institute of Architects). 
10. El encargo se produjo en 1962 y Eero Saarinen habia fallecido el 1 de septiembre de 1961, a los 51 años.

11. Dark steel pavilion designed for export 1966: 86

12. En esos momentos operaban aún bajo el nombre de Eero Saarinen and Associates. No fue hasta el 1 de septiembre de 1966 cuando el estudio cambió su nombre a Kevin Roche John Dinkeloo and Associates.

13. Estudio de arquitectura con sede en Londres desde 1947. El arquitecto encargado de los proyectos de Darlington era Fello Atkinson, uno de los tres socios iniciales. En la actualidad, el estudio sigue funcionando a nivel internacional.

14. La correspondencia de John Dinkeloo se ha consultado en la Biblioteca de Manuscritos y Archivos de la Universidad de Yale, MS 1884, caja 363 (John Dinkeloo Papers).

15. El proyecto de ambas fábricas se encuentra en las mismas cajas del Archivo MS 1884. Muchas veces es dificil diferenciar qué documentos se asocian a una o a otra y, en cuanto a la correspondencia, habitualmente se hablaba de ambos proyectos en una misma misiva.

16. La carga de trabajo del estudio en esos años era muy intensa. Por un lado estaban terminando las obras incompletas de Saarinen (el CBS, el Dulles o el Stiles and Morse entre otros) y por otro, trabajaban duro para mantener el estudio adelante tras la pérdida de su maestro. En 1964, además, ya contaban con algunos de sus encargos más importantes: el museo de Oakland, la Fundación Ford, el Instituto Richard C. Lee o el Instituto de Tecnología de Rochester.

17. Dark steel pavilion designed for export 1966: 82

18. Íb.: 86 .

19. Recuérdese que el primer edificio construido con este tipo de material en todo el mundo fue el Deere and Company Administrative Center en Molline, Illinois, de Eero Saarinen (1963). La utilización de juntas de neopreno estructurales en el muro cortina también es una innovación del estudio de Saarinen que lo utilizó por primera vez en el General Motors Technical Center en Warren, Michigan (1956).

20. John Dinkeloo comienza a trabajar para Eero Saarinen en 1951 y en 1956 ya era socio en Eero Saarinen \& Associates. Su papel en la investigación de materiales es reconocido por la profesión desde el principio y él mismo se encargó de divulgarlo a través de conferencias en universidades o congresos de ingeniería fundamentalmente. También publicó artículos en revistas periódicas, ver Dinkeloo 1962 o Dinkeloo 1964.

21. Un módulo de 30x60 pies equivale a $9,14 \mathrm{~m} \times$ $18,28 \mathrm{~m}(1$ pie $=30,48 \mathrm{~cm} ; 1$ pulgada $=2,54$ $\mathrm{cm})$. A lo largo del artículo se decide mantener el sistema imperial en vez del métrico ya que se considera que se entienden mejor los módulos empleados y las relaciones entre ellos.

22. El arquitecto paisajista Dan Kiley (1912-2004) fue un colaborador habitual de Kevin Roche John Dinkeloo and Associates, una relación profesional que heredaron de Eero Saarinen. KRJDA y Kiley realizaron algunos de los edificios más emblemáticos de la arquitectura americana de los años sesenta, destacando entre todos la Fundación Ford (1968) y el Museo de Oakland (1969).

23. Como curiosidad, cada máquina tenía anclada una placa de metal en la que se mostraba su coste, de manera que cada trabajador era consciente del precio de la máquina de la que era responsable.

24. El precio por metro cuadrado ascendió a 11,83 $\$$ del momento, excluyendo el coste del suelo y la maquinaria industrial. (Dark steel pavilion designed for export 1966: 86)

25. Dark steel pavilion designed for export 1966.

26. N.T. 1967: 12-25.

27. Aunque Kevin Roche nació en Irlanda se convirtió en ciudadano americano en 1963.
28. "The Cummins Engine Company's plant at Darlington is perhaps the most professionally conceived building put up in Britain since the war (N.T. 1967): 12

29. Ver Wagner 1968 ó Kevin Roche 1970

30. Ref. web 3:

http://www.krjda.com/Sites/CumminsSubInfo1.html (visitado 10 de mar. 2016)

31. "This plant affords every worker a view of the countryside, and by having parking on the roof brought each worker within a few steps of his job, and eliminated the need for acres of asphalt parking lots." Carta de J. Irwin Miller al AIA fechada a 2 de octubre de 1992 (Washington D.C: American Institute of Architects).

32. Extracto de una entrevista con la autora el 24 de marzo de 2016 en el estudio de KRJDA, Hamden, CT.

33. "Altogether, it is one of the best possible places to work - and to spend a good portion of a productive life. And it is a contribution to its environment." Productive elegance for industry 1975: 126

34. Como curiosidad, este número fue el último que la revista Architectural Forum publicó en esa primera etapa. AAVV 1974.

35. "The fascination with glass and the extreme functional demands of the manufacturing plant, so prominent her, bring back memories of early moderns: Behren's AEG turbine factory in Berlin (1908-1909); Gropius and Meyer's Fagus factory (1911); or their model factory at the Werkbund exposition (1914); not to mention the factories of Albert Kahn in the U.S. (e.g. Ford Motor Company in Dearborn, 1917) or Owen Williams' Boots factory at Beeston Nolts [sic] in England of 1932."Walesboro Component Plant, Cummin Engine Company. 1974. Architectural Forum 2: 49.

36. Von Eckardt. 1971: 45.

37. Éste es el motivo por el que fue elegida para formar parte del patrimonio arquitectónico protegido de Inglaterra. Baillieu 1992: 7.

38. El artículo muestra fotografias de Paul White tomadas en el año en el que se escribió el texto, es decir, 27 años después de su construcción. Winter 1992: 38.

39. "Alan Richardson, facilities manager for all the Cummins UK plants said the company was sorry to see the building go, that it had been a great success (...), he described the building as maintenance free." Winter 1992: 38.

40. El organismo encargado de seleccionar estos edificios es, en su idioma original, el "Department of National Heritage." Baillieu 1992: 7.

41. El artículo afirma que el edificio está abandonado y a la venta, en una zona que ha perdido interés desde el punto de vista industrial. Cummins in peril 1998.

42. Kevin Roche John Dinkeloo and Associates MS 1884, caja 384. Biblioteca de Manuscritos y Archivos, Universidad de Yale.

43. Carta de J. Irwin Miller al AIA fechada a 2 de octubre de 1992 (Washington D.C: American Institute of Architects).

\section{Bibliografia}

LIBROS

Dal Co, Francesco. 1985. Kevin Roche. Nueva York: Rizzoli International Publications Inc.

Futagawa, Yukio. 1975. Kevin Roche John Dinkeloo and Associates 1962-1975. Tokyo: A.D.A. Edita Tokyo Co., Ltd.

Harwood, Elain. 2000. England. A guide to post-war listed buildings. Londres: Ellipsis London Ltd.

Pelkonen, Eeva-Liisa. 2011. Kevin Roche: Architecture as environment. Yale: Yale University Press. 


\section{ARTÍCULOS}

Baillieu, Amanda. 1992. Three post-war buildings chosen for listing by Heritage dept. Building Design. 2 Octubre: 7.

Cummins Engine Co. Offices and factory. Darlington. 1967. Architectural Design 7: 360-361.

Cummins in peril. 1998. Architectural Review 1221: 18.

Dark steel pavilion designed for export. 1966. Architectural Forum 3: 82-87.

Dinkeloo, John. 1962. The steel will weather naturally. Architectural Record 131: 147-150.

Dinkeloo, John. 1964. Bold and Direct Using Metal in a Strong Basic Way. Architectural Record 133: 135-142.

Glueck, Grace. 1981. No Headline. The New York Times. 16 de junio.

Huxtable, Ada Louise. 1970. Creations of 3 top architects shown. The New York Times. 30 Septiembre.

Kevin Roche. 1970. Kenchiku Bunka 25: 71-90.

Medici of the west. 1974. Architectural Forum 2: 4647.

N.T.: 1967. Made in Mid-Atlantic. Architectural Review 845: $12-25$.

Productive elegance for industry. Component plant for Cummins Engine Company. 1975. Architectural Record 1: 126-130.

Rosie, George. 1969. Factory Design. Design Journal 250: 26-39.

Stephens, Suzanne. 1974. Savvy about steel, game with glass. Progressive Architecture 9: 78-83.

Technical study: weathering steels 2 . Case study 1 , Cummins Engine Factory, Darlington. 1972. Architects' Journal 45: 1075-1079.

The anatomy of the factory. Cummins Engine Co. Darlington. 1968. Architectural Design 11: 544.

Von Eckardt, Wolf. 1971. You can't see the foyer for the trees. Horizon: 45.

Wagner, Walter F. 1968. New directions. The work of Kevin Roche John Dinkeloo and Associates. Architectural Record 5: 145-168.

Walesboro Component Plant, Cummins Engine Company. 1974. Architectural Forum 2: 48-51.

Weathering steels become loadbearing. 1967. Progressive Architecture 9: 148-150.

Winter, John. 1992. Steel appeal Architects' Journal: 36-47.

Nota a la bibliografia: Cuando no ha sido posible encontrar el autor de un artículo, se ha colocado el título del texto en la posición del nombre del autor con el fin de facilitar la lectura y la identificación de citas al lector.

\section{Referencias web}

Ref. web 1: Modernism in Bartholomew County, Indiana, from 1942. United States Department of Interior, National Park Service. https://www.nps.gov/nhl/learn/specialstudies/ModernismBartholomewIN.pdf (visitado 26 feb. 2014).

Ref. web 2: De la Fuente, Ana. 2015. Columbus, Eero Saarinen e Irwin Miller. Origen y desarrollo de Architecture Program. http://www.cosasdearquitectos.com/2015/07/columbus-eero-saarinen-e-irwin-miller-origen-y-desarrollo-delarchitecture-program/ (visitado 1 abr. 2016).

Ref. web 3: www.krjda.com

Fecha final recepción articulos: 24/04/2016

Fecha aceptación

19/06/2016

Artículo sometido a revisión por dos revisores independientes por el método doble ciego. 